\title{
Etat d'infestation, connaissance endogène et approche systématique des espèces du genre Striga au Burkina Faso
}

\author{
I. J. BOUSSIM ${ }^{1}$, D. YONLI ${ }^{2 *}$, S. GUINKO ${ }^{1}$ et G. SALLE $^{3}$ \\ ${ }^{1}$ Université de Ouagadougou, Unité de formation et de recherche en sciences de la vie et de la terre, B.P. 7021, \\ Ouagadougou, Burkina Faso. \\ ${ }^{2}$ Institut de l'Environnement et de Recherches Agricoles, 04 B.P. 8645 Ouagadougou 04, Burkina Faso. \\ ${ }^{3}$ Université Pierre et Marie Curie, Laboratoire de Cytologie Expérimentale et de Morphogenèse Végétale, 4 \\ Place Jessieu, Bât. N2, Case courrier 150, 752552 Paris cedex 05, France. \\ *Auteur correspondant, E-mail : d.yonli313@gmail.com
}

\section{RESUME}

Des prospections de terrain ont été réalisées dans les trois domaines phytogéographiques du Burkina Faso pour inventorier les espèces de Striga, identifier leurs plantes hôtes et déterminer leur distribution géographique sur le territoire. Des enquêtes ont également été effectuées auprès des chercheurs, des agriculteurs et d'autres populations rurales pour appréhender leurs appréciations sur l'utilisation et les stratégies locales de lutte contre ces plantes parasites. Les prospections ont montré que le territoire est infesté par au moins 13 espèces de Striga, parmi lesquelles la présence de $S$. forbesii a été révélée pour la première fois au Burkina Faso. L'incidence économique des 13 espèces de Striga varie selon les conditions agro-écologiques locales et le type de culture pratiqué. $S$. hermonthica, $S$. gesnerioides et $S$. aspera sont dans l'ordre décroissant de virulence, les trois principales plantes parasites qui attaquent les cultures vivrières du pays. Des méthodes paysannes ont été recensées et les plus courantes sont l'arrachage et le sarclage manuels. Parmi les espèces de Striga inventoriées, seule $S$. hermonthica est connue pour ses vertus thérapeutiques et est aussi utilisée dans des systèmes tinctoriaux.

(C) 2011 International Formulae Group. All rights reserved.

Mots clés: Striga spp., infestation, distribution géographique, hôtes, perception endogène, Burkina Faso.

\section{INTRODUCTION}

Les espèces du genre Striga sont des plantes parasites épirhizes qui sont incapables d'accomplir leur cycle biologique en absence d'une angiosperme herbacée (Raynal-Roques, 1993) à l'exception d'une seule espèce, $S$. euphrasioides (Ramaiah et al., 1983). Elles constituent l'une des principales contraintes agronomiques en Afrique Sub-saharienne. En effet, 20 à 40 millions d'hectares de terres sont infestés par des espèces de Striga (Thalouarn et Fer, 1993; Ransom et
Odhiambo, 1995) et les pertes annuelles en rendement grains des cultures hôtes sont évaluées à 10,7 millions de tonnes (Gressel et al., 2004). Des technologies de lutte contre les espèces de Striga ont été développées, mais prises isolément, la plupart se sont révélées inefficaces.

La plupart des activités antérieures de recherche sur les plantes parasites herbaceés au Burkina Faso ont été focalisées sur Striga hermonthica et $S$. gesnerioides (Traoré et al., 1996 ; Ouédraogo et al., 2000; Traoré et al., 
2001 ; Tignegré, 2010 ; Yonli et al., 2010) dont l'effet dépressif sur leurs cultures hôtes s'amplifie suite aux aléas climatiques et à la dégradation des sols observés durant ces dernières décennies. L'écologie nous rappelle d'une part que ces deux espèces ont d'abord évolué dans le milieu naturel (Raynal-Roques, 1993 ) et d'autre part que des espèces inoffensives dans une contrée donnée peuvent être des ravageurs redoutables dans d'autres. En effet, toutes les espèces de Striga ont d'abord existé dans la nature avant que certaines s'adaptent progressivement aux cultures (Sallé et al., 1995). De ce fait, il y'a lieu de s'inquiéter que les espèces dites mineures aujourd'hui ou même celles qui l'ont jamais été encore sur les cultures, ne deviennent majeures ou parasites à l'avenir et que celles qui sont dans le milieu naturel ne s'adaptent aux espèces cultivées. Cette inquiétude est d'autant justifiée que $S$. asiatica qui ne parasite que des graminées sauvages au Burkina Faso, constitue ailleurs une contrainte agronomique pour les cultures céréalières (Mhele, 1987; Visser, 1987; Chidley et Drennan, 1987 ; Frisen et Korwar, 1991). En effet dans un territoire voisin du Burkina Faso à savoir le Togo, cette plante parasite sévit dans les champs de maïs (Zea mays L.) dont la production est affectée dans les champs fortement infestés (Agbobli et Huguenin, 1987). Ainsi, la connaissance parfaite de l'état d'infestation par les différentes espèces de Striga au Burkina Faso permettrait un meilleur suivi de la distribution géographique et de la dynamique de population de chaque espèce ainsi que son agressivité vis-à-vis de ses hôtes en particulier les cultures hôtes.

La présente étude a pour objectifs d'inventorier les espèces de Striga, de déterminer leur écologie sur l'ensemble du territoire burkinabé et de requérir les avis des paysans.

\section{MATERIEL ET METHODES Matériel}

Le Burkina Faso, d'une superficie de $274122 \mathrm{~km}^{2}$, est situé en Afrique Occidentale entre les latitudes $9^{\circ} 02^{\prime}$ et $15^{\circ} 05^{\prime} \mathrm{N}$ et les longitudes $02^{\circ} 02^{\prime}$ et $05^{\circ} 03^{\prime} \mathrm{E}$. Le climat est de type sahélien au nord dont la pluviométrie est de $300-500 \mathrm{~mm}$ avec trois mois de pluies et soudanien du centre au sud dont la pluviométrie se situe entre 700 et $1100 \mathrm{~mm}$ avec cinq mois de pluies (Guinko, 1984).

Des prospections saisonnières de terrain ont été effectuées à véhicule de 1990 à 2009 dans les quatre domaines phytogéographiques du Burkina Faso afin d'inventorier les espèces de Striga et autres plantes parasites. Les quatre domaines phytogéographiques (Figure 1) ont été décrits par Guinko (1984):

- Domaine phytogéographique Nord sahélien: la saison sèche est longue et le déficit pluviométrique est très important, où s'étend une steppe arbustive parfois arborée. Les espèces caractéristiques de ce secteur sont des épineux ;

- Domaine phytogéographique Sud sahélien : situé entre $13^{\text {ème }}$ et $14^{\text {ème }}$ parallèles nord, avec une pluviométrie moyenne annuelle de $650 \mathrm{~mm}$, est caractérisé par des espèces sahéliennes et sub-sahéliennes ;

- Domaine phytogéographique Nord soudanien : est le plus densément peuplé. Il est soumis à de nombreux défrichements ainsi qu'à la coupe abusive du bois. Il est caractérisé d'une savane arborée dominée par des espèces protégées telle que Vitellaria paradoxa ;

- Domaine phytogéographique Sud soudanien : situé à l'extrême sud et sud-ouest du Burkina Faso, avec une pluviométrie moyenne annuelle de $1100 \mathrm{~mm}$. Il est le mieux fourni en forêts claires et en savanes avec une strate arborée presque continue.

Sur le terrain, une presse a été utilisée pour conserver les plants à identification douteuse.

\section{Méthodes}

\section{Prospections des champs et jachères}

Les prospections ont été effectuées suivant des transects choisis en fonction des axes routiers praticables à véhicules durant la saison pluvieuse et traversant de nombreuses 


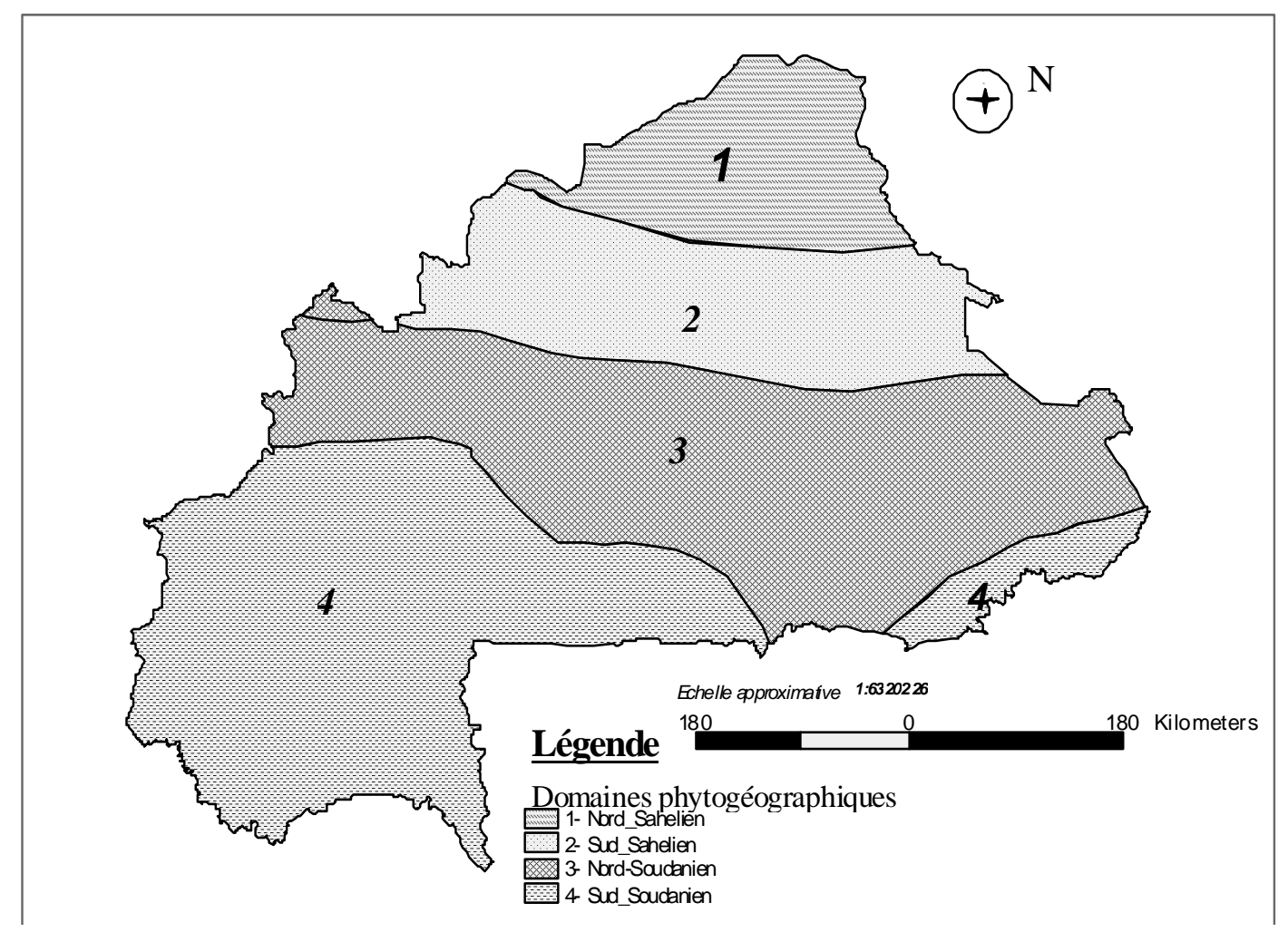

Figure 1:Grandes subdivisions phytogéographiques du Burkina Faso (1,2,3,4) (Guinko,1984).

zones agricoles dans chacun des quatre domaines phytogéographiques. Sur chaque transect, des arrêts de prospection sont observés dans 2-3 champs et la jachère ; situés à chaque distance de $30-40 \mathrm{~km}$. La prospection a consisté à traverser un champ ou une jachère deux fois de façon perpendiculaire et éventuellement faire le tour de la périphérie si le champ ou la jachère est de petites dimensions (< 1,5 ha). Sur les axes de parcours, les différentes espèces parasites du genre Striga ont été recensées. Celles dont l'identification est douteuse ont été récoltées et mises sous presse. La détermination de certaines espèces a été faite à l'aide des flores de Hutchinson et Dalziel (1954), de Hutchinson (1964), de Berhaut $(1967,1988)$ et de l'Afrique du Nord (Maire et Quézel, 1961) et d'autres par le laboratoire de Pierre et Marie Curie (France). Enquêtes

Une enquête a été réalisée auprès des chercheurs travaillant sur les mauvaises herbes, en particulier sur les plantes parasites, des agriculteurs et des populations rurales afin de compléter la liste des espèces recensées à partir de nos prospections et obtenir l'appréciation des enquêtés sur les espèces de Striga et les stratégies locales de lutte.

\section{RESULTATS ET DISCUSSION Etat d'infestation}

Treize (13) espèces de Striga ont pu être inventoriées sur l'ensemble du territoire Burkinabé dont les caractéristiques botaniques ont déjà été décrites par Musselman (1987), Paker et Riches (1993) et Kamel et al. (2001). Ce sont: Striga asiatica var. coccinea, $S$. asiatica var. lutea, S. aspera, S. baumannii, $S$. bilabiata, $S$. brachycalyx, $S$. forbesii, $S$. gesnerioides, S. hermonthica, S. klingii, $S$. linearifolia, S. macrantha, et $S$. passargei. Parmi ces 13 espèces, $S$. forbesii a été inventoriée pour la première fois au Burkina Faso. La présence des 12 autres espèces avait 
été rapportée par Toutain (1973), Guinko (1984), Hoffmann (1987) et Raynal-Roques (1995); ce qui confirme la véracité de l'identification des espèces collectées.

Les 13 espèces de Striga recensées au Burkina Faso représentent $76 \%$ du nombre total des espèces de Striga inventoriées en Afrique de l'ouest (Toutain, 1973 ; RaynalRoques, 1995) et $38 \%$ des taxons en Afrique. Parmi les 13 espèces de Striga, 12 parasitent au moins 44 espèces Graminées appartenant à 28 genres répartis en deux familles (Tableau 1) tandis que quatre parasitent au moins 13 espèces Légumineuses appartenant à 9 genres répartis en quatre familles (Tableau 2).

Des prospections similaires révèlent que la flore du Burkina Faso comporte au moins 25 espèces de Phanérogames parasites réparties dans 6 familles: Cuscutaceae, Lauraceae, Loranthaceae, Olacaceae, Santalaceae, Scrophulariaceae et dans 13 genres (Boussim, 2002). La famille des Scrophulariaceae est la mieux représentée avec 18 espèces appartenant à six genres notamment Alectra, Buchnera, Micrargeria, Rhamphicarpa, Sopubia et Striga. Dans cette famille, le genre Striga est le plus dominant (Boussim, 2002) à travers le territoire.

\section{Ecologie}

- Striga asiatica (L.) O. Ktze.

Synonymies: Buchnera asiatica L., S. lutea Lour., S. hirsuta Benth. Striga asiatica a été observée sous deux formes notamment la forme naine à fleurs rouge sang correspondant à la variété coccinea et la forme plus grande, à fleurs jaune pâle, la variété lutea.

Striga asiatica a été recensée dans les sites d'observation localisés dans les domaines phytogéographiques Sud soudanien et Nord soudanien où elle forme dans le milieu naturel de petites populations homogènes. Son aire d'occupation et sa densité dans les zones infestées sont encore faibles bien qu'elle ait une large distribution dans ces deux domaines phytogéographiques du pays jusqu'à la lisière du Sahel. Elle a été fréquemment relevée sur des sols gravillonnaires pauvres (S. asiatica var. lutea) ou cuirassés pouvant être gorgés d'eau en saison pluvieuse ( $S$. asiatica var. coccinea). $S$. asiatica est une plante hémiparasite annuelle. Elle attaque, au Burkina Faso, exclusivement des graminées sauvages. En effet, nos prospections nous ont révélé neuf Graminées sauvages servant d'hôtes à $S$. asiatica (Tableau 1). S. asiatica var. coccinea parasite surtout Ctenium villosum, Elionurus elegans, Microchloa indica, Loudetia togoensis, Tripogon minimis tandis que $S$. asiatica var. lutea infeste essentiellement A. fastigiatus, Andropogon pseudapricus, Loudetia togoensis, L. simplex, Schizachyrium exile et Sporobolus festivus. En Afrique de l'Ouest, $S$. asiatica hypothèque la production du maïs au Togo (Parker et Riches, 1993). Elle cause ailleurs d'importantes pertes de rendement aux cultures de sorgho (Sorghum spp.) et maïs (Zea mays) qui sont ses hôtes privilégiés et affecte légèrement le mil (Pennisetum spp.), le riz (Oryza sativa Starch) et la canne à sucre (Saccharum officinarum L.) (Parker et Riches, 1993).

\section{- Striga aspera (Willd.) Benth.}

Synonymies: Striga aspera var. filiformis Benth., Euphrasia aspera Willd. Striga aspera est l'une des espèces de Striga la plus fréquente dans les régions soudaniennes et soudano-sahéliennes du Burkina Faso. C'est une plante héliophile qui se développe en pieds isolés peu ramifiés ou en colonies dans le milieu naturel. Elle infeste les différents types de sol et est souvent rencontrée dans les zones marécageuses. $S$. aspera parasite au Burkina Faso au moins 12 espèces de Graminées (Tableau 1). Parmi les espèces attaquées, six principales cultures du pays font partie de ses hôtes potentiels à savoir le maïs, le sorgho, le mil, le fonio, l'arachide et le niébé. De faibles infestations de la plante parasite ont été relevées dans les champs de ces différentes cultures exceptés quelques champs de maïs où des infestations moyennes ont pu être observées à l'Ouest du pays (région soudanienne). Toutefois, $S$. aspera ne constitue pas une contrainte agronomique majeure pour l'agriculture burkinabé. Cependant, elle menace 
sévèrement la production du maïs dans certaines localités du Nigeria, du Cameroun, de la Côte d'Ivoire et de l'Ethiopie et celle du riz en Côte d'Ivoire et au Sénégal (Parker et Riches, 1993).

- Striga baumannii Engl.

Striga baumannii est une espèce discrète au Burkina Faso. Nous l'avons récolté essentiellement dans six localités situées au Sud du pays dont la pluviométrie annuelle est généralement supérieure à $1000 \mathrm{~mm}$ : Zabré, Niangoloko, Banfora, Batié, Gaoua, Léo. La tige florifère a une courte durée de vie. La plante passe la plus grande partie de l'année sous la forme de souche souterraine en latence. Seul le morphotype de saison sèche a $\mathrm{pu}$ être récolté entre avril et mai. Guinko (1984) avait aussi récolté un morphotype similaire dans la zone de Léo durant la même période de l'année. Le morphotype de saison humide n'a pas pu être observé à cause de sa courte durée de vie et surtout du fait qu'il apparaît à une période où les prospections de terrain sont difficiles en raison de la forte pluviosité et de l'impraticabilité des voies. De ce fait, ses plantes hôtes n'ont pas pu être identifiées durant nos prospections. RaynalRoques (1985) rapporte que les plantes hôtes de $S$. baumannii ne sont pas connues. Néanmoins, elle souligne l'existence des connexions racines avec d'autres plantes qui appartiendraient à des familles des Asclepiadaceae, Commelinaceae, Compositae et Papilionaceae. La double apparition de $S$. baumannii notamment en saison pluvieuse et en saison sèche, élargie très probablement le nombre de ses plantes hôtes. Toutefois, $S$. baumannii ne représente aucune menace pour les plantes cultivées au Burkina Faso. Elle est largement répandue en Afrique au Nord de l'équateur à savoir du Kenya à la Sierra Leone où elle pousse dans les savanes soudaniennes sur les plateaux rocheux (600-2000 m d'altitude), à climat humide (pluviométrie $\geq$ 1000 m) (Raynal-Roques, 1987, 1991).

- Striga bilabiata (Thunb.) O. Ktze.

Synonymies: Buchnera bilabiata Thunb., Striga thunbergii Benth. Striga bilabiata se rencontre dans les régions recevant plus de $800 \mathrm{~mm}$ d'eau par an. Nous avons récolté la sous-espèce jargeri dans sept localités notamment à Pama, Pô, Nazinga, Léo, Bobo Dioulasso, Banfora et Gaoua, dans des savanes soudaniennes sur des souches de sept espèces vivaces (Tableau 1) qui donnent de nombreuses repousses après le passage des feux de brousse. Boudet et Lebrun (1986) et Hoffmann (1994) rapportent que Striga bilabiata est rencontrée dans les savanes soudaniennes où elle est représentée par 3 sous-espèces : rowlandii, barteri et jargeri. Les 2 premières sous-espèces se développent essentiellement dans le milieu naturel où elles occupent des biotopes différents. Elles poussent pendant la saison pluvieuse en pieds isolés et présentent chacune une grande spécificité parasitaire. S. bilabiata subsp. Rowlandii se développe sous l'ombrage des arbres et arbustes tandis que $S$. bilabiata subsp. Barteri pousse dans les prairies bien ensoleillées. Toutes les trois sous-espèces parasitent des graminées vivaces.

\section{- Striga brachycalyx Skan.}

Synonymie : Striga warneckei Engl. Ex Skan. Striga brachycalyx s'observe surtout dans les savanes soudaniennes et, dans une moindre mesure, soudano-sahéliennes. Elle est fréquente dans la moitié Sud du Burkina Faso. Elle pousse sur des sols gravillonnaires, argileux ou argilo-sableux. Dans la zone Sud soudanienne, son attaque à des plantes pérennes telles que Andropogon ascinodis, Schizacirium sanguineum amplifie son parasitisme. C'est une espèce qui s'avère du point de vue agronomique dangereuse du fait de l'existence probable de 2 morphotypes, lui permettant de coloniser des biotopes écologiquement différents. En effet, un morphotype de 30-40 cm de hauteur, très peu ramifié est rencontré dans les savanes soudano-sahéliennes et soudaniennes où il parasite des graminées annelles. Un autre, de $60-80 \mathrm{~cm}$ de hauteur, très ramifié, pousse dans les milieux humides et infeste des Graminées pérennes. Nos prospections nous ont permis d'inventorier treize Poaceae, une Cyperaceae et deux Légumineuses comme hôtes à $S$. brachycalyx. Généralement inféodée au milieu 
naturel, Striga brachycalyx a été exceptionnellement observée au Mali sur le système racinaire de quelques pieds de sorgho et de mil (Hoffmann, 1994). Au Burkina Faso, l'abondance de cette espèce dans le milieu naturel requiert une vigilance particulière car des modifications et variations adaptatives pourraient survenir et rendre l'espèce nuisible à certaines cultures.

\section{- Striga forbesii Benth.}

Striga forbesii est très rare au Burkina Faso. La plante parasite pousse dans des zones humides à forte précipitation, plus de $800 \mathrm{~mm}$ de pluie par an. Elle a été observée sur des sols lourds abritant la culture de maïs uniquement dans le domaine phytogéographique Sud soudanien dans trois localités: la vallée du Kou dans des parcelles irriguées expérimentales de contre saison, Bala et Douna. Sa densité dans les parcelles était très faible et ne pouvait pas de ce fait constituer un facteur limitant pour la culture hôte. En revanche, S. forbesii affecte les cultures de sorgho et de maïs dans certaines localités au Zimbabwé et en Tanzanie, du riz en Côte d'Ivoire et de la canne à sucre en Somalie (Parker et Riches, 1993). Elle parasite également des Graminées sauvages telles que Setaria sphacelata et Echinochloa pyramidalis (Parker et Riches, 1993).

- Striga gesnerioides (Willd.) Vatke

Synonymies : Buchnera gesnerioides

Willd., Striga orobanchoides Benth. S. gesnerioides a une aire de développement qui couvre pratiquement tout le territoire burkinabé depuis les régions Sud jusqu'à l'extrême Nord. Les fortes densités sont relevées sur les sols gravillonnaires, sableux et sablo-limoneux. Elle est largement répandue en Afrique et s'avère l'espèce de Striga commune à toutes les régions africaines (Parker et Riches, 1993). S. gesnerioides est l'une des deux espèces de Striga les mieux connues au Burkina Faso par l'importance horizontale et verticale des cultures attaquées. Après $S$. hermonthica, c'est $S$. gesnerioides qui est citée par les agriculteurs comme la seconde mauvaise herbe parasite la plus destructive. Au Burkina Faso, elle est inféodée au niébé (Vigna unguiculata (L.) Walp.) dont les pertes de productions enregistrées sont estimées entre $30 \%$ (Aggarwal et Ouédraogo, 1989) et 44,2\% (Muleba et al., 1997). En Afrique de l'Ouest le niébé est la principale culture attaquée et des pertes de rendement pouvant atteindre jusqu'à $100 \%$ ont été signalées dans certaines zones de la sous-région ouest africaine (Emechebe et al., 1983). S. gesnerioides comporte des races physiologiques intraspécifiques vis-à-vis du niébé. Ces races ne peuvent pas être distinguées à partir des caractères morphologiques ou phénotypiques. A l'aide de la biotechnologie, trois races dénommées "Race 1", "Race 5" et "Kp" ont été identifiées au Burkina Faso (Tignegré, 2010). S. gesnerioides parasite aussi d'autres cultures notamment le tabac en Afrique du Sud (Parker et Riches, 1993), la tomate en Afrique du Sud et en Ethiopie (Parker et Riches, 1993). Au Burkina Faso, huit espèces fourragères appartenant aux familles des Convolvulaceae et Fabaceae sont parasitées (Tableau 2) dans les jachères et le milieu naturel. Parker et Riches (1993) ont signalé d'autres espèces légumineuses hôtes appartenant à cinq familles: Acanthaceae, Euphorbiacae, Leguminosae, Pedaliaceae et Solanaceae.

\section{- Striga hermonthica (Del.) Benth.}

Synonymies : Buchnera hermonthica

Del., Striga senegalensis Benth. Striga hermonthica est essentiellement une espèce africaine à distribution intertropicale, sauf la bande forestière équatoriale (Bâ, 1984). Le territoire du Burkina Faso étant inclus dans cette bande climatique, S. hermonthica s'y rencontre partout, souvent en fortes populations luxuriantes. Les fortes infestations s'observent dans les champs situés dans les domaines phytogéographiques Sud sahélien et Nord soudanien correspondant aux régions du plateau central, Est et Sud du pays. Elle se développe sur tous les types de sols, mais montre une préférence pour les sols sableux et gravillonnaires, épuisés par la culture continue durant plusieurs années. Les observations de Traoré et Yonli (2001) à l'Est du pays ont révélé que les sols sableux et gravillonnaires 
situés en haut de pente, les sols légers pauvres en argile et en éléments nutritifs sont les plus infestés par $S$. hermonthica. En revanche durant nos prospections, elle a pu être observée dans des zones marécageuses sur des sols limoneux et hydromorphes. Cette observation suggère la capacité d'adaptation de la plante parasite aux différents biotopes. $S$. hermonthica est l'adventice la plus redoutée par les agriculteurs burkinabé du fait qu'elle constitue un fléau sans précédent pour les cultures de sorgho, mil et maïs qui sont les principales céréales vivrières. En effet, les pertes dans la production de sorgho dues à $S$. hermonthica ont été évaluées au centre du pays entre $7 \%$ et $41 \%$ (Zombré et Nikiéma, 1992 ) et à l'Est entre $28 \%$ et $55 \%$ (Traoré et Yonli, 2001). Elle parasite sporadiquement le riz, le fonio (Digitaria exilis (Kippist) Stapf) au Burkina Faso et attaque des cultures légumineuses (sésame, arachide) dans d'autres contrées de l'Afrique de l'Ouest (Parker et Riches, 1993). Les pertes monétaires dues à $S$. hermonthica sur le sorgho, le mil et le maïs ont été estimées en Afrique sub-Saharienne respectivement à 89,82 et 140 millions de dollars US (M'Boob, 1986). Vingt six (26) Graminées sauvages (Tableau 1) et une Scrophulariaceae notamment Alectra vogelii (Tableau 2) ont été inventoriées durant nos prospections comme hôtes dans les jachères.

\section{- Striga klingii (Engl.) Skan.}

Synonymie : Buchnera klingii Engl. Striga klingii est rare au Burkina Faso. C'est un hémiparasite qui pousse sur des sols alluvionnaires riches et profonds des bas fonds où elle parasite des Graminées sauvages vivaces parmi lesquelles quatre ont pu être recensées (Tableau 1). Nous l'avons toujours observé en fin de la saison pluvieuse dans quatre localités réparties dans le domaine phytogéographique Sud soudanien: à Zabré, dans la vallée du fleuve Nazinon ; à Nazinga, dans la région de Pô ; à Boromo, dans la vallée du Mouhoun ; à Diéfoula, dans la région de Niangoloko dans la vallée de Comoé. De ce fait, S. klingii n'a aucune incidence économique sur l'agriculture au Burkina Faso.

\section{- Striga linearifolia (Schum. \& Thonn.) Hepper}

Synonymie : Striga bilabiata subsp. linearifolia (Schum. \& Thonn.) Mohamed. Striga linearifolia est très rare au Burkina Faso. Sa présence avait été signalée sur le territoire Burkinabé (Toutain, 1973) mais aucun herbier du pays ne disposait d'un spécimen récolté avant notre étude. Cette espèce a été relevée à faible densité sur des sols sablo-argileux, sols alluvio-limoneux et hydromorphes. Elle n'a été observée pour la première fois durant nos prospections de terrain qu'en septembre 2009 dans deux localités situées dans le domaine phytogéographique Sud soudanien: à Sangou et à Zoaga, dans le département de Zabré, province du Boulgou. Dans les deux localités, elle avait pour hôte une graminée pérenne (Sporobolus pyramidalis P. Beauv.) et pourrait probablement infester d'autres Graminées pérennes sauvages. Cette espèce n'a aucune incidence agronomique au Burkina Faso. Kamal et al. (2001) ont considéré $S$. linearifolia comme la sixième sous-espèce de S. bilabiata et avaient souligné sa présence en Afrique dans les savanes herbeuses humides.

\section{- Striga macrantha (Benth.) Benth.}

Synonymie : Buchnera macrantha Benth. Striga macrantha a été observée en pieds isolés ou en petites colonies pratiquement aux mêmes endroits que $S$. klingii dans la zone soudanienne mais jamais dans la zone sahélienne. Cette espèce pousse sur des sols riches et profonds des bas-fonds et a été relevée dans six sites notamment dans la forêt classée de Toessé à Yako, à la vallée du Nazinon, dans la réserve de la mare aux hippopotames dans la région de Bobo Dioulasso, à Léo, à Banfora et à Gaoua. Dans ces sites, son hôte principal était Andropogon gayanus et pourrait probablement parasiter d'autres Graminées pérennes sauvages.

\section{- Striga passargei Engl.}

Striga passargei est très répandue au Burkina, elle a été observée sur presque tout le territoire. C'est une espèce précoce par rapport aux autres espèces de Striga recensées au Burkina Faso. En effet, dès fin juillet, des 
pieds de $S$. passargei fleuris sont observés dans le domaine phytogéographique Sud soudanien. Nos prospections révèlent que $S$. passargei est un parasite mineur au Burkina Faso, elle infeste faiblement les champs de mil dont la production n'est pas affectée. Elle colonise plus les jachères où les graminées annuelles constituent ses hôtes parmi lesquels 11 ont pu être inventoriés (Tableau 1). Le fonio, le maïs, le mil et le sorgho sont les plantes cultivées qui sont occasionnellement parasitées (Parkinson, 1985 ; Paré, 1993). Hoffmann (1994) souligne que le maïs était fortement endommagé par S. passargei au Nord du Nigeria.

\section{Clé d'identification des espèces de Striga inventoriées au Burkina Faso}

Espèces dont le calice a 4 à 5 côtes $(1$ côte par lobe du calice) : Pentapleurae Plante scabre, haute de $50 \mathrm{~cm}$ à $1 \mathrm{~m}$, feuilles larges, calice long de plus de $6 \mathrm{~cm}$. Les fleurs, dont la largeur des lèvres est comprise entre 1 et 2 $\mathrm{cm}$, sont roses. A la base de chaque fleur se trouve une bractée aussi large (1 à $3 \mathrm{~mm}$ ) que le calice qu'elle cache. De gros cils blancs aux bords des bractées et des lobes du calice. Le tube de la corolle > $10 \mathrm{~mm}$ de long, est coudé dans sa partie inférieure, au niveau du sommet du calice. S. hermonthica.

Plante haute de plus de $50 \mathrm{~cm}$, à aspect plus frêle que $S$. hermonthica. Les fleurs, dont la largeur des lèvres est environ $1 \mathrm{~cm}$, sont roses. Le tube de la corolle, long de 1 à 1,5 $\mathrm{cm}$, est un peu plus droit et se courbe dans le dernier tiers de celle-ci. Les bractées larges de 1 à $2 \mathrm{~mm}$ sont dépourvues d'une frange de cils. ..S. aspera.

Plante haute de 15 à $30 \mathrm{~cm}$, fleurs sessiles de couleur blanc crème à jaune, parfois rose pâle. Les bractées sont plus longues que le calice..................... .. passargei.

Plante haute de 15 à $30 \mathrm{~cm}$, vert pâle ou violacée, pauvre en chlorophylle; feuilles réduites à des écailles. Les fleurs, mauve pâle souvent blanches, dont les lèvres sont larges de $5 \mathrm{~mm}$ environ, à tube étroit et coudé, sont terminées par des lobes étalés. L’inflorescence en épi occupe presque toute la hauteur de la tige au-dessus du sol. Tube de la corolle 8-14 $\mathrm{mm}$ de long. .................. gesnerioides.

Plante haute de 15 à $30 \mathrm{~cm}$, à tiges et feuilles pubescentes, rugueuses au touché. Tube de la corolle pubescent, calice $>4 \mathrm{~mm}$ de long......S. bilabiata. Plantes pubescente, dont les poils sont orientés vers le bas ......S. linearifolia.

Espèces dont le calice a 10 à 14 côtes (essentiellement 2 côtes par lobe du calice). Plante haute de $15-30 \mathrm{~cm}$. Petites fleurs jaunes ou rouge vif, toujours jaune pâle à l'extérieur de la corolle. .........S. asiatica.

- forme naine à fleurs rouge sang............ S. asiatica var. coccinea ;

- forme plus grande, à fleurs jaune pâle...... S. asiatica var. lutea Plante glabre, à tige grêle, feuilles réduites en écailles, fleurs petites à tube étroit..........S. baumannii. Espèces dont le calice a 10 ou plus de côtes : Polypleurae Plante annuelle, haute de $50 \mathrm{~cm}$ environ; les feuilles larges de plus de $1,5 \mathrm{~cm}$ sont dentées et plus petites que les entrenœuds. Les fleurs avec des lèvres larges de 1 à $2 \mathrm{~cm}$ sont pâles, rose saumon, parfois blanches. Le tube corollaire, long de 10-13 $\mathrm{mm}$, est coudé juste en dessous des lobes de la corolle. Les côtés du calice sont un peu poilues..................S. forbesii.

Plante grêle, à petites fleurs roses. La largeur de la lèvre inférieure mesure 4 à 5 $\mathrm{mm}$. Le calice a une hauteur 1,5 à $3 \mathrm{~mm}$. A la base de chaque fleur, une petite feuille (bractée) nettement plus réduite que les feuilles situées plus bas sur la tige................. brachycalyx.

Plante haute de 0,5 à $1,5 \mathrm{~m}$. Tige peu ramifiée, ligneuse. Grandes feuilles dentées pouvant atteindre $10 \mathrm{~cm}$ de long et $1 \mathrm{~cm}$ de largueur, sont plus longues que les entrenœuds. Inflorescences en épis terminaux. Grandes fleurs blanches, tube corollaire 17-24 mm de long. .............S. macrantha.

Plante haute de 0,5 à $1 \mathrm{~m}$. Tige peu ou pas ramifiée. Feuilles linéaires, grossièrement dentées ou non. Inflorescence en épi terminal, compacte. Fleurs blanches, mauves, à tube court, peu dégagé de l'épi. Les tiges, les 
feuilles, les bractées et le calice sont densément couverts de poils durs, dressés.............S. klingii.

\section{Méthodes de lutte et utilisation locales}

Nos enquêtes ont révélé que les paysans ne connaissent que le parasitisme de deux espèces de Striga: $S$. hermonthica et $S$. gesnerioides. Les autres espèces de Scrophulariaceae sont considérées comme des adventices ordinaires. Ainsi, ils développent des initiatives pour contrôler le parasitisme de ces deux espèces et plus particulièrement $S$. hermonthica. A travers nos prospections de terrain et enquête, les mesures préventives sont méconnues mais sept méthodes locales censées curatives ont été dénombrées : l'arrachage et le sarclage manuels, l'application de la fumure organique dans les champs, l'utilisation des cosses de néré, l'épandage des résidus des amandes de karité, la rotation culturale et la mise en jachère. En plus de ces méthodes paysannes, le semis précoce, le buttage, l'utilisation de la cendre de Striga, de la poudre d'écorce d'Acacia gourmaensis Bark et de la graisse de chèvre noire ont été cités à l'Est du pays comme recettes locales dans la lutte contre le Striga (Traoré et Yonli, 2001). Emechebe et al. (2004) ont dénombré 15 stratégies locales de lutte contre le Striga au Nigeria dont les plus courantes étaient aussi l'arrachage, le sarclage manuel et la fertilisation des champs avec la fumure organique.

Parmi les espèces de Striga recensées au Burkina Faso, seule S. hermonthica est utilisée dans la teinture et dans les traitements thérapeutiques des populations. En effet, la plante de $S$. hermonthica constitue une composante importante dans le traitement traditionnel des calculs salivaires, urinaires et vésicaux, du paludisme, du choléra, de la coqueluche, du diabète, du tabagisme, de l'alcoolisme et de la goutte. Dans la région Est du Burkina Faso, S. hermonthica est une plante médicinale très utilisée en pharmacopée pour soigner une dizaine de maladies et à d'autres fins qui s'intègrent parfaitement aux réalités socio-culturelles des populations (Traoré et Yonli, 2001). Au Sénégal, les belles fleurs rose violet de la plante parasite sont exploitées par les fleuristes qui les vendent emballées dans des sachets plastiques transparents au même titre que les roses. Les propriétés utilitaires de Striga suggèrent que les méthodes de lutte élaborées ne doivent pas viser son éradication totale mais plutôt la réduction de l'infestation dans les champs afin que l'effet dépressif de leur parasitisme soit en dessous du seuil de nuisibilité.

Tableau 1 : Les graminées relevées comme plantes hôtes des espèces de Striga au Burkina Faso.

\begin{tabular}{|c|c|c|c|c|c|c|c|c|c|c|}
\hline \multirow[b]{2}{*}{ Plantes hôtes de la famille des Poaceae } & \multicolumn{10}{|c|}{ Espèces de Striga } \\
\hline & $\begin{array}{l}\text { S. } \\
\text { asi }\end{array}$ & $\begin{array}{l}\text { S. } \\
\text { asp }\end{array}$ & & $\begin{array}{l}\text { S. } \\
\text { bra }\end{array}$ & $\begin{array}{l}\text { S. } \\
\text { for }\end{array}$ & $\begin{array}{l}\text { S. } \\
\text { her }\end{array}$ & $\begin{array}{l}\text { S. } \\
\text { ges }\end{array}$ & $\begin{array}{ll}\text { S. } & \text { S. } \\
\text { kli } & \text { lin }\end{array}$ & $\begin{array}{l}\text { S. } \\
\text { mac }\end{array}$ & $\begin{array}{l}\text { S. } \\
\text { pas }\end{array}$ \\
\hline Andropogon ascinodis C.B.CI & & $\mathrm{x}$ & $\mathrm{x}$ & $\mathrm{x}$ & & $\mathrm{x}$ & & & & \\
\hline Andropogon fascitigiatus Sw. & $\mathrm{x}$ & $\mathrm{x}$ & $\mathrm{x}$ & & & $\mathrm{x}$ & & & & $\mathrm{x}$ \\
\hline Andropogon gayanus Kunth. & & & $\mathrm{x}$ & & & & & $\mathrm{x}$ & $\mathrm{x}$ & $\mathrm{x}$ \\
\hline Andropogon pseudapricus Stapf. & $\mathrm{x}$ & $\mathrm{x}$ & $\mathrm{x}$ & $\mathrm{x}$ & & $\mathrm{x}$ & & & & $\mathrm{x}$ \\
\hline Aristida adsencionis L. & & $\mathrm{x}$ & & & & $\mathrm{x}$ & & & & $\mathrm{x}$ \\
\hline Aristida kerstingii Pilger. & & $\mathrm{x}$ & $\mathrm{x}$ & & & & & & & $\mathrm{x}$ \\
\hline Brachiaria disticophylla (Trin.) Stapf. & & $\mathrm{x}$ & & & & $\mathrm{x}$ & & & & \\
\hline Brachiaria lata C.E. Hubbard & & $\mathrm{x}$ & & & & $\mathrm{x}$ & & & & \\
\hline Cenchrus biflorus Roxb. & & $\mathrm{x}$ & & & & & & & & \\
\hline
\end{tabular}


Ctenium villosum Berhaut

$\mathrm{X} \quad \mathrm{X}$

Cymbopogon gigantus Chiov.

$\mathrm{X}$

Cymbopogon schoenanthus (L.) Spreng.

Cynodon dactylon (L.) Pers.

Dactyloctenium aegyptium Wiid.

Digitaria gayana (Kunth.) Stapf

Digitaria horizontalis Willd.

Diheteropogon amplectens W.D. Clayton

Elionurus elegans Kunth.

Eragrostis aspera (Jacq.) Nees

Eragrostis tremula (Hochst)

Eragrostis turgida (Schumach.) Dewild.

Eleusine indica (L.) Gaertn.

Hackelochloa granularis (L.) O. Ktze

Loudetia simplex (Nees) C.E. Hubbard

Loudetia togoensis (Pilger) C.E. Hubbard

Microchloa indica (L.) P. Beauv.

$\mathrm{X} \quad \mathrm{X}$

$\mathrm{X}$

$\mathrm{X} \quad \mathrm{X}$

$\mathrm{X}$

X $\quad \mathrm{X} \quad \mathrm{X} \quad \mathrm{X}$

X $\quad \mathrm{X}$

X

$\mathrm{X}-\mathrm{X}$

$x$

X

$\mathrm{X} \quad \mathrm{X} \quad \mathrm{X}$

X $\mathrm{X} \quad \mathrm{X} \quad \mathrm{X}$

$\mathrm{X} \quad \mathrm{X}$

$\mathrm{X}$

$\mathrm{X}$

Pennisetum americanum K. Schum.

$\mathrm{X}$

$\mathrm{X}$

$\mathrm{X} \quad \mathrm{X}$

$\mathrm{X}$

Schizachyrium exile (Hochst.) Pilger

Schizachyrium sanguineum Alton

Schoenepheldia gracilis Kunth.

Setaria pallide-fusca Stapf et C.E.

Hubbard

Sorghum bicolor (L.) Moench

Sporobolus festivus Hochst.

Sporobolus pyramidalis P. Beauv.

Zea mays L.

$\begin{array}{lll} & & \mathrm{X} \\ & & \mathrm{X} \\ & & \mathrm{X} \\ \mathrm{X} & \mathrm{X} \\ \mathrm{X} & \mathrm{X} \\ & & \mathrm{X} \\ \mathrm{X} & \mathrm{X} \\ & \mathrm{X} & \mathrm{X} \\ \mathrm{X} & \mathrm{X} & \\ & & \mathrm{X} \\ \mathrm{X} & \mathrm{X} & \mathrm{X} \\ \mathrm{X} & & \mathrm{X} \\ \mathrm{X} & \mathrm{X} & \\ & & \end{array}$

X X

Plantes hôtes de la famille des

Cyperacea

Bulbostylis barbata C.C.CI. 
Tableau 2 : Les espèces légumineuses relevées comme plantes hôtes des espèces de Striga au Burkina Faso.

\begin{tabular}{|c|c|c|c|c|c|c|c|c|c|}
\hline \multirow[b]{2}{*}{ Plantes hôtes } & \multicolumn{9}{|c|}{ Espèces de Striga } \\
\hline & $\begin{array}{l}\text { S. } \\
\text { asi }\end{array}$ & $\begin{array}{ll}\text { S. } & \text { S. } \\
\text { asp } & \text { bil }\end{array}$ & $\begin{array}{l}\text { S. } \\
\text { bra }\end{array}$ & $\begin{array}{l}S . \\
\text { for }\end{array}$ & $\begin{array}{l}\text { S. } \\
\text { ges }\end{array}$ & $\begin{array}{ll}\text { S. } & \text { S. } \\
\text { her } & \text { kli }\end{array}$ & $\begin{array}{l}\text { S. } \\
\text { lin }\end{array}$ & $\begin{array}{l}\text { S. } \\
\text { mac }\end{array}$ & $\begin{array}{l}\text { S. } \\
\text { pas }\end{array}$ \\
\hline \multicolumn{10}{|l|}{ Famille des Convolvulaceae } \\
\hline Ipomoea eriocarpa $\mathrm{R} . \mathrm{Br}$. & & & & & $\mathrm{x}$ & & & & \\
\hline Ipomoea pes-carpaea (L.) & & & & & $\mathrm{x}$ & & & & \\
\hline Ipomoea vagans Bak. & & & & & $\mathrm{x}$ & & & & \\
\hline \multicolumn{10}{|l|}{ Famille des Fabaceae } \\
\hline Alysicarpus ovalifolius J. Léonard & & & $\mathrm{x}$ & & $\mathrm{x}$ & & & & \\
\hline Arachis hypogaea $\mathrm{L}$. & & $\mathrm{x}$ & & & & $\mathrm{x}$ & & & \\
\hline Indigofera tintoria $\mathrm{L}$. & & $\mathrm{x}$ & & & $\mathrm{x}$ & & & & \\
\hline Tephrosia bracteolata Guil. Et Perr. & & $\mathrm{x}$ & & & $\mathrm{x}$ & & & & \\
\hline Tephrosia pedicelata Bakl. & & & & & $\mathrm{x}$ & & & & \\
\hline Vigna unguiculata (L.) Walp. & & $\mathrm{x}$ & & & $\mathrm{x}$ & & & & \\
\hline \multicolumn{10}{|l|}{ Vigna sunterranea (L.) Verdc. } \\
\hline Zornia glochidiata Reichb. ex DC. & & $\mathrm{x}$ & & & $\mathrm{x}$ & & & & \\
\hline \multicolumn{10}{|l|}{ famille des Rubiaceae } \\
\hline Spermacoce radiata (DC.) Hiern. & & & $\mathrm{x}$ & & & & & & \\
\hline \multicolumn{10}{|l|}{ Famille des Scrophulariaceae } \\
\hline Alectra vogelii Benth. & & & & & & $\mathrm{x}$ & & & \\
\hline
\end{tabular}

\section{Conclusion}

La présence d'une espèce au Burkina Faso, Striga forbesii, non indiquée dans la littérature a été inventoriée pour la première fois. Trois espèces, $S$. aspera, S. gesnerioides et $S$. hermonthica ont été observées sans difficulté en raison de leur abondance dans la végétation et / ou de leur interférence avec les cultures. Par contre, S. asiatica var. lutea, $S$. forbesii, $S$. klingii, $S$. linearifolia et $S$. macrantha ont été retrouvées avec beaucoup de difficultés en raison de leur rareté et de leur discrétion. Les résultats de nos prospections de terrain et enquêtes auprès des populations révèlent une bonne implantation $\mathrm{du}$ parasitisme phanérogamique sur le territoire burkinabé et par extension dans les régions à climat soudanien.

Sur les 13 texa de Striga présents au Burkina Faso, deux espèces notamment $S$. gesnerioides et $S$. hermonthica constituent une contrainte biotique majeure pour l'agriculture et trois autres à savoir $S$. aspera, $S$. forbesii et $S$. passargei sont des parasites mineurs pour les cultures. Au Burkina Faso, les espèces de Striga ne sont pas les seules Scrophulariaceae parasites épirhizes qui attaquent les cultures, Alectra vogelii, Buchnera hispida et Rhamphicarpa fistulosa ont fréquemment été relevées dans les champs et méritent de ce fait une attention particulière.

\section{REMERCIEMENTS}

Nous exprimons notre profonde gratitude à la Fondation Internationale pour la Science (FIS), l'Université Pierre et Marie Curie en France, la Coopération française au Burkina Faso et le projet INCO-DC pour avoir financé cette étude. Nous remercions également l'Université de Ouagadougou au 
Burkina Faso pour les facilités qui nous ont été accordées pour la conduite des activités.

\section{REFERENCES}

Agbobli CA, Huguenin B. 1987. Evaluation agronomique du problème du parasitisme du Striga asiatica sur le maïs dans le Sud Togo. In Parasitic Flowering Plants, Weber HChr., Forstreuter W (eds). F.R.G: Marburg ; 11-26.

Aggarwal VD, Ouédraogo JT. 1989. Estimation of cowpea loss from Striga infestation. Trop. Agric., 66: 9192.

Bâ AT. 1984. Morphology, anatomy and ultrastructure of some parasitic species of the genus Striga (Scrophulariaceae). In Striga Biology and Control, Ayensu ES, Doggett H, Keynes RD, Marton-Lefèvre, Musselman LJ, Parker C, Pickering A (eds). ICSU Press: Paris; 47-58.

Berhaut J. 1967. Flore du Sénégal. Clairafrique : Dakar.

Berhaut J. 1988. Flore Illustrée du Sénégal. Clairafrique: Dakar.

Boussim IJ. 2002. Les phanérogames parasites du Burkina Faso : inventaire, taxonomie, écologie et quelques aspects de leur biologie. Cas particulier des Loranthaceae parasites du karité. Thèse d'Etat, université de Ouagadougou, 291 p.

Chidley VL, Drennan DSH. 1987. Effect on sorghum root residue on Striga asiatica (L) Kuntze infection (Scrophulariaceae). In Parasitic Flowering Plants, Weber HChr, Forstreuter W (eds). F.R.G: Marburg; 829-831.

Emechebe AM, Leleji O, Salako EA. 1983. Control of root parasitic weeds in cowpea and groundnut. IAR Symposium on Striga and its control, 23 may, IAR Samaru, Nigeria.

Emechebe AM, Ellis-Jones J, Schulz S, Chikoye D, Douthwaite B, Kureh I, Tarawali G, Hussaini MA, Kormawa P, Sanni A. 2004. Farmers' perception of the Striga problem and its control in northern Nigeria. Expl. Agric., 40: 215-232.

Friesen GH, Korwar GR. 1991. Effect of phenolic acids and mixed cropping on Striga asiatica infestations in Sorghum. In Proceedings of the Fifth International
Symposium on Parasitic Weeds, Ransom JK, Musselman LJ, Worsham AD, Parker C (eds). CMMYT: Nairobi; 10-13.

Guinko S. 1984. Végétation de la haute Volta. Thèse de Docteur ès Sciences Naturelles, Université De Bordeaux III, France, UER Aménagement et ressources Naturelles. Département l'homme et son environnement T.I et T.II, 394 p.

Gressel J, Hanafi A, Head G, Marasas W, Obilana AB, Ochanda J, Souissi T, Tzotzos G. 2004. Major heretofore intractable biotic constraints to African food security that may be amenable to novel biotechnological solutions. Crop Protection, 23: 661-689.

Hutchinson J. 1964. The Genera of Flowering Plants (Angiospermae). In Dicotyledones. Clarendon: Oxford; $516 \mathrm{p}$.

Hutchinson J, Dalziel MD. 1954. Flora of West Tropical Africa (Vol 1. Part 1. 2nd ed). Crown agents for oversea governments and administrations, Millbank: London; 827 p.

Kamal IK, Musselman LJ, Riches CR. 2001. The genus Striga (Scrophulariaceae) in Africa. Ann. Missouri Bot. Gard., 88: 60103.

Maire R, Quézel P. 1961. Flore de l'Afrique $d u$ Nord (Maroc, Algérie, Tunisie, Tripolitaine, Cyrénaïque et Sahara) (Vol. 3) Edit. Paul Lechevalier: Paris (VIe).

M'Boob SS. 1986. A regional program for Striga control in West and Central Africa. In Proc. of the FAO/OUA All Africa Government Consultation on Striga Control, Robson TO, Broad HR (eds). Maroua, Cameroun; 190-194.

Mhehe GL. 1987. A novel chemical approach to the control of witchweed (Striga asiatica (L.) Kuntze) and other Striga spp. (Scrophulariaceae), In Parasitic Flowering Plants, Weber HChr, Forstreuter W (eds). FRG: Marburg; 563-574.

Muleba N, Ouédraogo JT, Tignegré JB. 1997. Cowpea yield losses attributed to Striga infestations. Journal of Agriculture Science, 129: 43-48.

Musselman LJ. 1987. Taxonomy of witchweeds. In Parasitic Weeds in 
Agriculture Striga (Vol 1), Musselman LJ (ed). CRC Press: Florida, USA; 317 p.

Ouédraogo O, Sallé G, Tuquet C, Bouillant ML, Bally R. 2000. Spécification de plantes faux-hôtes pour Striga hermonthica (Del.) Benth. (Scrophulariaceae). Etudes et Recherches Sahéliennes, 4-5: 81-86.

Parker C, Riches CR. 1993. Parasitic Weeds of the World: Biology and Control. Castlefield Press Limited: Kettering, Northants; 332 p.

Paré J. 1993. Aspects de la dynamique de la formation de la graine chez le Striga (Scrophulariaceae) parasite des céréales tropicales. Thèse de doctorat d'Etat, UPMC/ Paris VI, 210 p.

Raynal-Roques A. 1985. An usual biology for a parasitic plant. Haustorium, $\mathrm{n}^{\circ} 15$.

Raynal-Roques A. 1987. The genus Striga (Scrophulariaceae) in Western and Central Africa- a survey. In Proceedings of the fourth International Symposium on Parasitic Flowering plants: Parasitic Flowering plants, Weber WChr, Forstreuter W (ed), Philipps-Universität; 675-689

Raynal-Roques A. 1991. Diversification in the genus Striga. In Proceedings Fifth International Symposium on parasitic weeds, Ransom JK, Musselman LJ, Worsham AD, Parker C, (eds). Nairobi, Kenya; 251-261.

Raynal-Roques A. 1993. Contribution à la connaissance de la biologie des Striga (Scrophulariaceae): types biologiques et phénologie. Bulletin Museum National Histoire Naturelle, Paris, 4è série, 15, section B, Adansonia, ${ }^{\circ}$ 1-4: 3-31.

Sallé G, Raynal-Roques A, Tuquet C. 1995. Un fléau en Afrique, les Striga. Vie Science, 12: 27-46.

Tignegré JB. 2010. Genetic Study of Cowpea (Vigna unguiculata (L.) Walp Resistance to Striga gesneriodes (Willd.) Vatke) in Burkina Faso. Ph. D. thesis in Plant Breeding, African Centre for Crop
Improvement, School of Agricultural Sciences and Agribusiness, Faculty of Science and Agriculture, University of KwaZulu-Natal, Republic of South Africa, $165 \mathrm{p}$.

Traoré D, Vincent C, Stewart RK. 1996. Association and synchrony of Smicronys guineanus and Smicronys umbrinus (Coleoptera : Curculionidea) and the parasitic weed Striga hermonthica (Del.) Benth. (Scrophulariaceae). Biological Control, 3: 307-315.

Traoré H, Hess DE, Hoffmann G, Son A, Sallé G. 2001. Use of hand-weeding and herbicides to control Striga hermonthica in Burkina Faso. African Crop Science Journal, 9: 645-653.

Traoré H, Yonli D. 2001. Striga et autres adventices : perception paysanne et inventaire des méthodes endogènes de lutte dans l'Est du Burkina Faso. Science et Technique, Sciences Naturelles et Agronomie, 25(1): 46-59.

Visser JH. 1987. The susceptibility of some sugar cane cultivar to witchweed, Striga asiatica (L.) Kuntze. In Parasitic Flowering Plants, Weber HChr, Forstreuter W (eds). FRG: Marburg; 789795.

Yonli D, Traoré H, Sérémé P, Hess DE, Sankara P. 2010. Pathogenicity of Fusarium isolates to Striga hermonthica in Burkina Faso. Pakistan Journal of Biological Sciences, 13: 201-208.

Zombré PN, Nikiéma S. 1992. Importance et effet de Striga hermonthica (Del.) Benth. sur la production du sorgho en zone Nord soudanienne du Burkina Faso: caq de Linonghin. Rev. Rés. Amélior. Prod. Agr. Milieu Aride, 4: 103-112. 